\title{
Hépatite alcoolique aiguë sévère
}

\section{Severe acute alcoholic hepatitis}

\section{E. Nguyen-Khac $\cdot$ V. Dejour $\cdot$ R. Sarba $\cdot$ T. Yzet $\cdot$ J. Turpin $\cdot$ D. Chatelain $\cdot$ I. Marcq $\cdot$ C. Chivot $\cdot$ J. Maizel $\cdot$ C.A. Papillon · C. Attencourt · H. Houchi}

Reçu le 3 août 2018; accepté le 15 octobre 2018

(C) SRLF et Lavoisier SAS 2018

Résumé Tout patient consommateur chronique et excessif d'alcool avec un ictère récent doit être évalué par le score de Maddrey à la recherche d'une hépatite alcoolique aiguë sévère. Les corticostéroïdes représentent le traitement de première ligne, associés à un soutien nutritionnel adapté et à une abstinence alcoolique. La combinaison corticostéroïdes plus $N$-acétylcystéine (perfusée pendant les cinq premiers jours) améliore la survie à court terme par rapport aux corticostéroïdes seuls, constituant une option thérapeutique de première ligne. La réponse au traitement est évaluée au septième jour par le modèle de Lille inférieur ou égal à 0,45 . Le pronostic des patients non répondeurs aux corticostéroïdes avec un modèle de Lille supérieur à 0,45 est sombre avec une survie de $23 \%$ à six mois. Pour des patients non répondeurs aux corticostéroïdes et sélectionnés, la transplantation hépatique précoce améliore significativement la survie à six mois et à long terme.

E. Nguyen-Khac $\cdot$ V. Dejour $\cdot$ R. Sarba $\cdot$ J. Turpin $\cdot$ H. Houchi

Service hépatogastroentérologie,

CHU Amiens-Picardie, Hôpital Sud,

F-80054 Amiens, France

E. Nguyen-Khac · I. Marcq · C.A. Papillon · H. Houchi Inserm U1247, groupe de recherche sur l'alcool et les pharmacodépendances (GRAP),

centre universitaire de recherche en santé (CURS),

CHU Amiens, université de Picardie Jules-Verne (UPJV),

F- 80054, France

T. Yzet $\cdot$ C. Chivot

Service de radiologie digestive, CHU Amiens-Picardie,

Hôpital Sud, F-80054 Amiens, France

D. Chatelain $\cdot$ C. Attencourt

Service d'anatomopathologie, CHU Amiens-Picardie,

Hôpital Sud, F-80054 Amiens, France

\section{J. Maizel $(\bowtie)$}

Service de réanimation médicale, CHU Amiens-Picardie, Hôpital Sud, F-80054 Amiens, France

e-mail : julien.maizel@u-picardie.fr
Mots clés Hépatite alcoolique $\cdot$ Alcool · Cirrhose · Biopsie foie $\cdot$ Corticostéroïdes $\cdot N$-acétylcystéine $\cdot$ Maddrey score : Lille model $\cdot$ MELD $\cdot$ ABIC score $\cdot$ GAHS

Abstract All the chronic and excessive consumers of alcohol with recent jaundice should be assessed using a Maddrey's score for severe acute alcoholic hepatitis. Corticosteroids are the first-line treatment associated with an appropriate nutritional support and alcohol abstinence. Corticosteroids plus $\mathrm{N}$-acetylcysteine combination improves short-term survival over corticosteroids alone, and could be proposed as a firstline therapy. The response to treatment is evaluated at the $7^{\text {th }}$ day of treatment, with the Lille model $\leq 0.45$. Prognostic of non-responders to corticosteroids with Lille model $>0.45$ is dramatically low with $23 \%$ survival at 6 months. Early liver transplantation in a selected group of patients with nonresponse to corticosteroids significantly improves at sixthmonth and is long-term survival.

Keywords Alcoholic hepatitis · Alcohol - Cirrhosis · liver biopsy $\cdot$ corticosteroids $\cdot \mathrm{N}$ acetylcystein $\cdot$ Maddrey score Lille model $\cdot$ MELD $\cdot$ ABIC score $\cdot$ GAHS

\section{Introduction}

La maladie alcoolique du foie (MAF) englobe un spectre de lésions hépatiques allant de la simple stéatose, touchant près de $90 \%$ de consommateurs chroniques et excessifs d'alcool, à l'apparition progressive d'une fibrose hépatique puis d'une cirrhose dans $20 \%$ des cas, avec ensuite son cortège de complications telles que l'hypertension portale, l'insuffisance hépatique et le carcinome hépatocellulaire [1]. Cette histoire naturelle est émaillée d'une lésion inflammatoire représentée par l'hépatite alcoolique, intimement liée à la poursuite de la consommation d'alcool. L'hépatite alcoolique a une présentation histologique caractéristique avec des foyers de nécrose hépatocytaire associés à une infiltration de polynucléaires neutrophiles, une ballonisation hépatocytaire et la présence 
de corps de Mallory (Fig. 1). L'expression clinique est habituellement peu symptomatique dans la phase de stéatohépatite lorsqu'il y a encore peu de fibrose hépatique et pas de cirrhose. Le pronostic est alors bon avec à l'arrêt de la consommation d'alcool une régression des lésions histologiques. Il n'y a pas de traitement spécifique hormis l'évaluation addictologique.

Inversement, il existe une forme sévère habituellement appelée hépatite alcoolique aiguë (HAA), se développant chez un patient ayant dans plus de $90 \%$ des cas une cirrhose sous-jacente constituée, soit déjà connue, soit découverte à l'occasion de l'épisode d'HAA. Cette forme est très symptomatique et se manifeste cliniquement par l'apparition rapide d'un ictère dans un contexte de consommation active d'alcool, de signes d'insuffisance hépatocellulaire et d'une encéphalopathie hépatique. L'HAA sévère engage le pronostic à court et moyen termes, avec une mortalité proche de $25 \%$ à un mois et de $50 \%$ à six mois [2]. En l'absence de traitement, la mortalité spontanée évaluée à 28 jours est comprise entre 34,9 et $44 \%$ [3]. L'incidence de l'HAA n'est pas bien connue en France.

\section{Physiopathologie}

Les mécanismes physiopathologiques de l'hépatite alcoolique sont complexes intriquant des mécanismes comme l'augmentation de la perméabilité de la barrière intestinale avec le passage dans la circulation portale puis dans le foie du lipopolysaccharide composant de la paroi des bactéries (LPS), l'activation de l'immunité innée du foie, l'infiltration hépatique par les PNN, la mort de l'hépatocyte par nécrose et/ou apoptose et la variabilité interindividuelle des facteurs génétiques. Plus récemment, le rôle de la dysbiose intestinale induite par l'alcool a été étudié avec un intérêt croissant.

\section{Dysbiose intestinale et augmentation de la perméabilité intestinale}

Le microbiote intestinal (MI) ou flore intestinale est composé de $10^{14}$ micro-organismes représentant dix fois plus que le nombre de cellules de l'organisme humain. On considère que chaque individu possède son propre MI, stabilisé dans sa composition dès les premières années de la vie. La consommation chronique d'alcool est responsable d'une modification du MI (dysbiose) avec chez l'animal une diminution des phyla Lactobacillus, Bacteroïdetes, Firmicutes, une augmentation des Proteobacteria ainsi qu'une diminution globale de la diversité du MI. Chez l'homme, la dysbiose existe aussi chez les patients alcooliques avec une diminution des Bacteroïdetes et une augmentation des Enterobacteriacae et des Proteobacteria [4]. Cette dysbiose pourrait expliquer des mécanismes de diminution de la couche de mucus participant à la protection des entérocytes, une diminution par certaines souches de microbactéries de la synthèse de protéines participant à la constitution des tight jonctions des entérocytes, induisant au final l'augmentation de la perméabilité intestinale, expliquant le passage plus fréquent des bactériémies dans la circulation portale arrivant ensuite dans le foie. Il a été démontré qu'une transplantation de microbiote de patients ayant une HAA à des souris germ free et soumises ensuite à un régime alimentaire de Lieber de Carli enrichi progressivement en alcool permettait de déclencher une inflammation du foie en comparaison à des souris témoins soumises au même régime, mais recevant une transplantation de MI d'un humain sans HAA [5]. La dysbiose intestinale liée à la consommation d'alcool est donc probablement impliquée dans les processus physiopathologiques de l'HAA.

\section{Métabolisme de l'alcool et stress oxydatif}

Le métabolisme de l'éthanol génère un stress oxydatif avec la production d'espèces réactives à l'oxygène (ROS). Ainsi, la

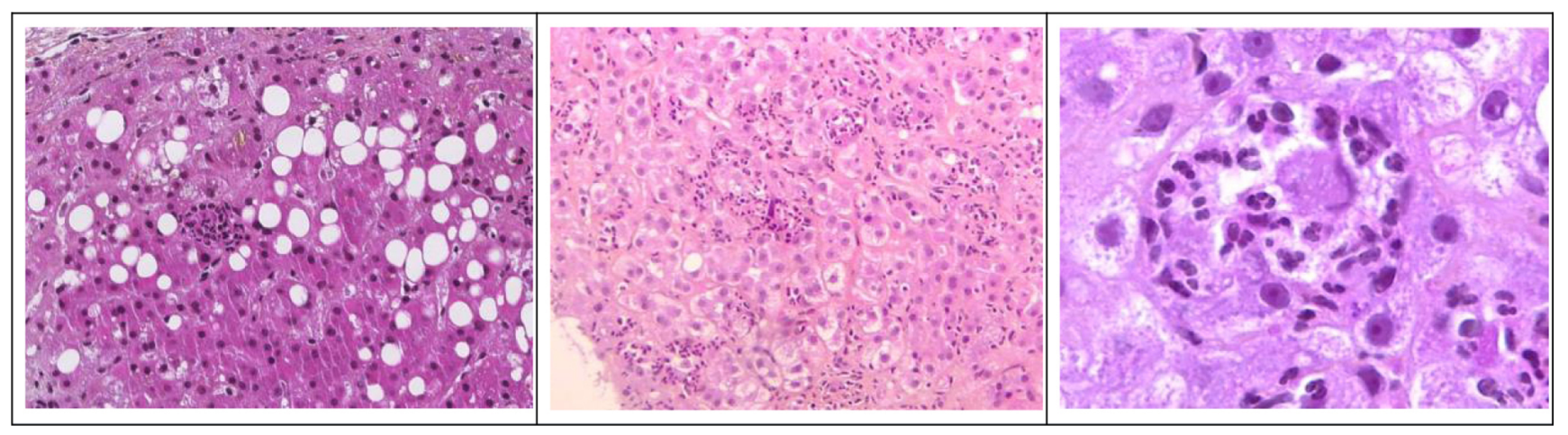

Fig. 1 Histologie hépatique en microscopie optique, coloration HES (hématoxyline éosine safran). Foyers d'hépatite alcoolique, corps de Mallory et stéatose macrovacuolaire (A). Hépatite alcoolique avec hépatocytes en nécrose ou apoptose entourés de polynucléaires neutrophiles, faible grossissement $\times 10$ (B). Fort grossissement $\times 40$ (C). Courtoisie : Pr Denis Chatelain, service anatomopathologie, CHU Amiens-Picardie 
toxicité cellulaire est due à l'acétaldéhyde qui est le principal métabolite de l'alcool produit presque exclusivement par l'alcool déshydrogénase cytosolique ( $\mathrm{ADH})$ des hépatocytes, et accessoirement par le cytochrome P450 dans le microsome, ou encore par la catalase dans les peroxysomes [6].

Les autres causes de la majoration du stress oxydatif sont la peroxydation lipidique sur un foie stéatosique et une diminution du glutathion mitochondrial et de la $S$-adénosine méthionine [7] largement consommés par la production concomitante de ROS. L'acétaldéhyde est ensuite transformé dans la mitochondrie en acétate qui donnera finalement en dehors de l'hépatocyte dans d'autres organes du dioxyde de carbone $\left(\mathrm{CO}_{2}\right)$.

Dans ce contexte d'alcoolisation chronique, la baisse du stock intramitochondrial de glutathion (GSH) est démontrée dans les modèles animaux, attribuée à un défaut de transport du GSH du cytosol vers la mitochondrie [8]. Ce déficit en GSH précède les lésions hépatiques chez la souris exposée à l'alcool [9]. Les conséquences sont une baisse des capacités antioxydantes hépatocytaires, le GSH mitochondrial étant un mécanisme majeur de détoxification de l'excès de peroxyde d'hydrogène $\left(\mathrm{H}_{2} \mathrm{O}_{2}\right)$ et des radicaux libres. Il a également été suggéré qu'une baisse du GSH mitochondrial pourrait rendre l'hépatocyte plus sensible au tumor necrosis factor- $\alpha$ (TNF$\alpha$ ) induisant la mort cellulaire [10].

\section{Processus inflammatoires}

Parallèlement, la cellule de Kupffer est stimulée [11] par les lipopolysaccharides (LPS/endotoxinémie), constituants principaux de la membrane externe des bacilles à Gram négatif, en provenance du tube digestif, dont la perméabilité est augmentée par les épisodes répétés de consommation importante d'alcool [12]. Le LPS circule avec sa protéine porteuse de la LPS binding protein (LBP) et active une cascade inflammatoire induite par la voie des récepteurs Toll intracellulaire et les récepteurs TLR4 à la surface de la cellule de Kupffer [9]. Son activation induit la production de ROS, de cytokines pro-inflammatoires et de TNF- $\alpha$. Des données suggèrent également une activation de la cellule de Kupffer par le complément C3 et C5 [13]. Le TNF- $\alpha$ induit des lésions hépatiques par l'intermédiaire de deux récepteurs membranaires TNFR1 et TNFR2. Le récepteur TNFR1 a un rôle prédominant, comme le montre l'absence de lésion hépatique chez la souris knock-out TNFR1 exposée à l'alcool. À l'inverse, l'activation de la cellule de Kupffer produit un effet cytoprotecteur via l'interleukine-6 (IL-6) et l'IL-10 [12]. Ces deux cytokines anti-inflammatoires activent le facteur de transcription STAT3 de l'hépatocyte, des cellules endothéliales et des macrophages, à l'origine de la transcription de facteurs anti-apoptotiques, antioxydants et pro-oncogéniques. La régulation entre les facteurs délétères pro-inflammatoires et protecteurs anti-inflammatoires reste à éclaircir [14].

L'infiltration par des PNN autour d'hépatocytes en cours de souffrance ou de mort cellulaire est l'image histologique caractéristique de l'hépatite alcoolique, attirés par chimiotactisme lié à une augmentation d'IL-8, de CXCL1 et d'IL-17, et aussi d'IL-1, de TNF- $\alpha$ et d'ostéopontine [14]. Cependant, le rôle exact des PNN n'est pas bien connu. Enfin, l'hépatite alcoolique reste une maladie multifactorielle, car, à durée et quantité de consommation d'alcool égales, tous les patients ne développeront pas la maladie, suggérant un terrain de vulnérabilité génétique [15]. Les données concernant les singles nucléotides polymorphismes (SNP) sont nombreuses [16], mais non concluantes. Les données sur l'adiponutrine (patatin-like phospholipase domain-containing protein 3 [PNPLA3]) semblent plus robustes. Chez des patients éthyliques chroniques, le polymorphisme rs738409 $\mathrm{C}>\mathrm{G}$ est associé à un risque accru de stéatose et de cirrhose en cas d'homozygotie G/G. Dans une série, la présence de l'allèle muté $\mathrm{G}$ était plus fréquente chez des patients ayant une hépatite alcoolique sévère par rapport à un groupe témoin sain et était associée à une survie plus courte.

\section{Diagnostic}

\section{Présentation clinicobiologique}

Cliniquement, le diagnostic d'une HAA est évoqué chez un patient ayant une consommation active et excessive d'alcool (jusqu'à en moyenne 150 à $200 \mathrm{~g} / \mathrm{j}$ dans les essais cliniques) et présentant un ictère d'installation récente associé à une insuffisance hépatique pouvant se traduire par des signes d'encéphalopathie hépatique (Fig. 2). Habituellement, le délai entre l'exposition à l'alcool et la survenue du tableau clinique aigu est considéré comme inférieur à trois mois. Cette présentation est accompagnée par des signes de décompensation hépatique comme l'ascite, parfois une hémorragie par hypertension portale, voire un syndrome hépatorénal. Une fièvre est possible, devant faire rechercher une complication infectieuse dont la fréquence a été rapportée jusqu'à $25 \%$ avant tout traitement [17]. Les infections spontanées du liquide d'ascite, les pneumopathies, les infections urinaires et les dermohypodermites sont les sites les plus fréquents à contrôler systématiquement. La présentation biologique comporte une élévation modérée des transaminases prédominant sur l'AST, une hyperbilirubinémie mixte et une altération de la coagulation avec en générale une baisse $\mathrm{du}$ taux de prothrombine. La seule information à tirer $d u$ rapport ASAT/ALAT supérieur à 1 est une orientation vers une cause alcoolique sans aucune autre valeur clinique ni pronostique. 


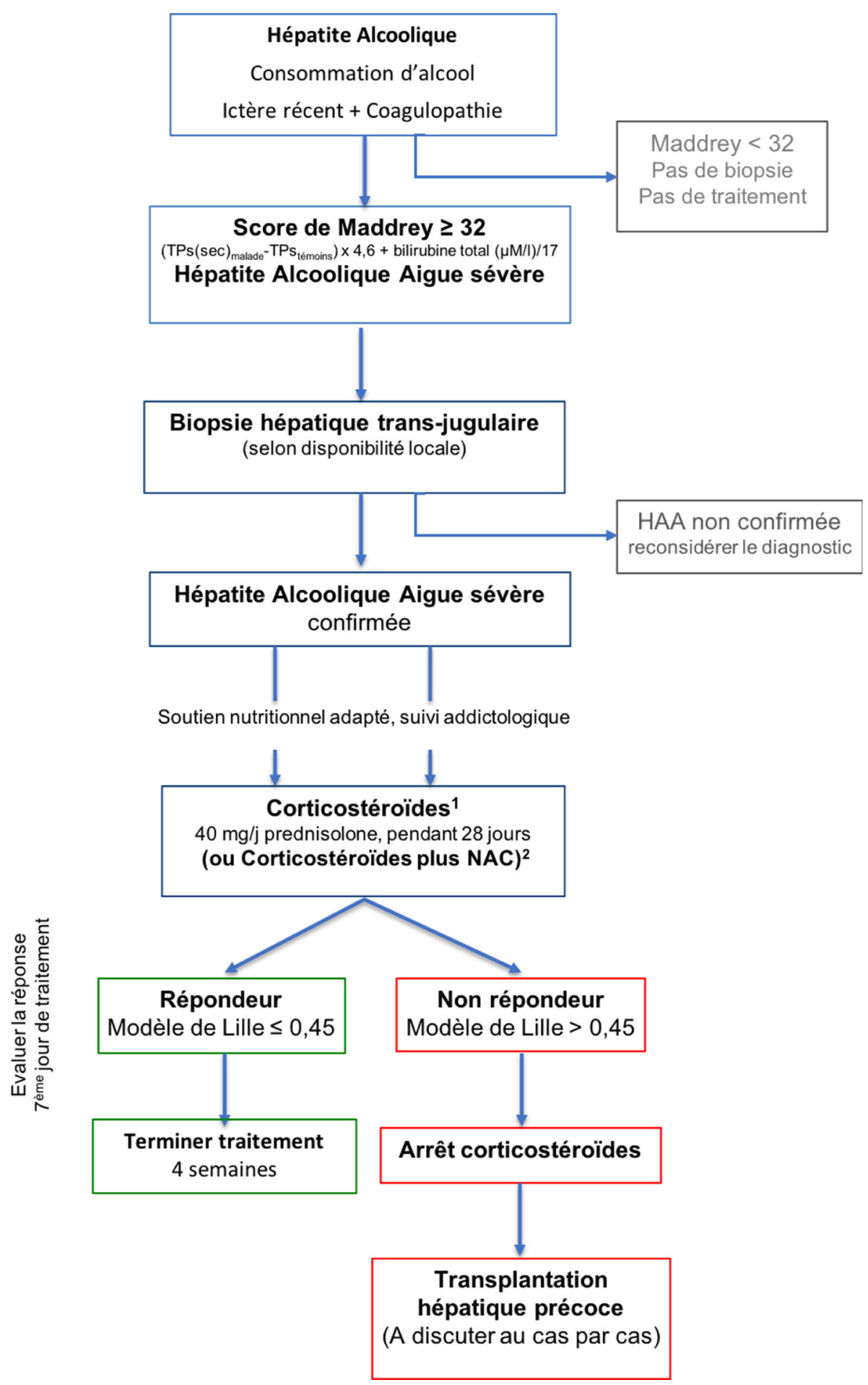

Fig. 2 Algorithme de prise en charge de l'hépatite alcoolique aiguë sévère. ${ }^{1}$ recommandation de grade A1 ; ${ }^{2}$ recommandation de grade B2 (guidelines EASL 2018 [1])

Le bilan complémentaire comportera une radiographie de thorax, une ponction d'ascite, un examen cytobactériologique des urines et des hémocultures en cas de fièvre. L'examen hépatique par une échographie est d'usage pour le bilan de l'hépatopathie, mais aussi pour le diagnostic différentiel du syndrome cholestatique, comme l'angiocholite, et du Budd Chiari en examen Doppler des veines sus-hépatiques. Enfin, une autre maladie hépatique (virale C ou B mais aussi A ou E) associée doit également être écartée. 


\section{Scores pronostiques}

La fonction discriminante de Maddrey (DF) permet d'identifier les patients ayant une hépatite alcoolique sévère. Publiée initialement en 1978 [18] et modifiée par Carithers et al. en 1989 [19], elle est calculée selon la formule : [temps de prothrombine en seconde (TPs) $\left.)_{\text {patient }}-\mathrm{TPs}_{\text {témoin }}\right]$ $\times 4,6+$ bilirubine totale $(\mu \mathrm{M}) / 17$. Dans une analyse rétrospective réalisée sur les groupes placebo de trois essais randomisés, le groupe des patients avec une DF supérieure ou égale à 32 avait une survie spontanée à 28 jours significativement plus basse de $67,6 \pm 7,1$ versus $93,2 \pm 3,8 \%$ pour les malades avec une DF inférieure à $32(p=0,003)$ [2]. Ce score est simple et facile à calculer au lit du patient.

D'autres scores plus récents, issus d'analyses multivariées, ont été investigués. Le score de MELD (Model for End-stage Liver Disease) décrit initialement pour évaluer le pronostic des patients cirrhotiques avec une hypertension portale a été évalué chez des patients avec une hépatite alcoolique [20,21]. Dans ces études, le MELD était associé à la mortalité des patients, avec des aires sous la courbe ROC (Receiver Operating Characteristic) comprises entre 0,76 à 0,97. Cependant, les cut-offs décrits étaient variables d'une série à l'autre pour définir la forme d'HAA sévère, entre 18 à 25, sans différence statistique avec la DF de Maddrey.

Le Glasgow Alcoholic Hepatitis Score (GAHS) comporte cinq variables indépendamment associées à la mortalité (âge, globules blancs, temps de prothrombine, bilirubine, urée) auxquelles des points croissants (un à trois points) sont alloués selon la gravité. Ce score a été construit sur une population de 241 patients alcooliques. Avec un GAHS supérieur ou égal à 9 , la survie était significativement inférieure à 28 et 84 jours par rapport au groupe ayant un GAHS inférieur à 9. La même équipe a ensuite testé l'impact d'un traitement par corticostéroïdes selon le GAHS. Les patients avec un GAHS supérieur ou égal à 9 avaient une meilleure survie lorsqu'ils étaient traités par corticostéroïdes par rapport à ceux traités par placebo, alors que les corticostéroïdes n'amélioraient pas la survie chez les patients avec un GAHS inférieur à 9 par rapport au placebo [22].

Plus récemment, le score ABIC (Âge, Bilirubine, INR [International Normalized Ratio] et Créatinine) a été décrit sur une population de 103 patients consommateurs excessifs d'alcool, avec une biopsie hépatique, et traités par corticostéroïdes dans $46 \%$ des cas [23]. Calculé par la formule $($ âge $\times 0,1)+($ bilirubine $\times 0,08)+(\operatorname{INR} \times 0,8)+($ créatinine $\times 0,3)$, le score définissait trois sous-groupes. Avec un score inférieur à 6,71 la survie était de $100 \%$ à 90 jours, alors qu'avec un score supérieur à 9 la survie n'était que de $25 \%$ à 90 jours. Le groupe intermédiaire, avec un score compris entre 6,71 à 9 , avait une survie à $70 \%(p<0,0001)$. L'aire sous la courbe ROC était significativement supérieure à celle de la DF de Maddrey dans la population de construction du modèle, mais le score ABIC était comparable aux scores MELD et GAHS dans la population de validation. De plus, dans une série indépendante, les patients, avec le meilleur pronostic avec un score $\mathrm{ABIC}$ inférieur à 6,71, avaient finalement une mortalité de $17,8 \%$ à 84 jours [24].

Ces nouveaux scores MELD, GAHS et ABIC manquent encore de validations indépendantes. Les récentes recommandations des sociétés américaine 2010 [25] et européenne 2018 [1] ont ainsi indiqué l'usage du score de Maddrey en première intention, en cas de présomption d'hépatite alcoolique, pour estimer le pronostic.

Récemment le concept d'Acute-on-Chronic Liver Failure (ACLF) a été développé dans l'étude CANONIC par le consortium Europeen EASL-CLIF (Chronic LIver Failure) pour mieux définir le pronostic des maladies chroniques du foie [26]. L'ACLF est définie par :

- une décompensation hépatique (ascite et/ou hémorragie digestive et/ou encéphalopathie hépatique) ;

- une défaillance d'organe (insuffisance hépatique : bilirubine $\geq 2 \mathrm{mg} / \mathrm{dl}$; insuffisance rénale : créatinine $\geq 2 \mathrm{mg} / \mathrm{dl}$ ou dialyse ; insuffisance cérébrale : encéphalopathie grades 3 à 4 de West Haven; insuffisance coagulopathie : INR $\geq 2,5$; insuffisance circulatoire : usage de vasoconstricteur systémique ; insuffisance respiratoire : $\mathrm{PaO}_{2} /$ $\mathrm{FiO}_{2}<200$ ou $\mathrm{SPO}_{2} / \mathrm{FiO}_{2}<214$ ou assistance ventilatoire);

- un pronostic vital engagé avec une mortalité à j28 supérieure à $15 \%$ [26].

Dans la population de l'étude CANONIC, $59 \%$ des patients n'avaient pas de défaillance d'organe (ACLF 0 : mortalité $4,5 \%$ à j28), $16 \%$ avaient une défaillance rénale ou une défaillance non rénale plus dysfonction rénale (créatinine entre 1,5 à 1,9 mg/dl) ou dysfonction cérébrale (EH de gardes 1 à 2) (ACLF 1 : mortalité $22 \%$ à j28), $11 \%$ avaient deux défaillances d'organes (ACLF 2 : mortalité $32 \%$ à j28) et $4 \%$ avaient trois à six défaillances d'organe (ACLF 3 : mortalité $78 \%$ à j28) [26]. Une première étude décrit l'incidence d'une ACLF chez 165 patients avec une HAA sévère dont $85 \%$ étaient traités par corticostéroïdes [27]. Au diagnostic de l'HAA, 47,9 \% des patients avaient une ACLF, avec un impact sur la survie $(15,7 \%$ étaient ACLF 1 : mortalité $30,8 \%$ à j28; $14,5 \%$ étaient ACLF 2 : mortalité $58,3 \%$ à j28 et $17,6 \%$ étaient ACLF $3:$ mortalité $72,4 \%$ à j28; $p<0,0001$ ), ainsi qu'une moins bonne réponse aux corticostéroïdes $(38,2$ versus $76,6 \% ; p<0,0001)$. Cependant, la valeur prédictive de la survie à j28 des patients avec une HAA était comparable entre la fonction dérivée de l'ACLF (https://www.clifresearch.com/ToolsCalculators. aspx) et le score de Maddrey avec un $\mathrm{C}$ index respectif de 0,68 (IC $95 \%:[0,59-0,76]$ ) et 0,64 (IC $95 \%:[0,56-0,73]$ ). Parmi les patients sans ACLF au diagnostic, $33 \%$ développaient une ACLF au cours du suivi de 168 jours. Au total, 
$65,5 \%$ des patients ayant une HAA ont ou développeront une ACLF. Le seul facteur prédictif indépendant en analyse multivariée de développer une ACLF de novo était l'infection au cours du suivi. Le développement d'une ACLF chez les patients avec une HAA est donc fréquent, mais la place de la fonction dérivée reste à déterminer.

\section{Biopsie hépatique et arguments histologiques}

La confirmation histologique de l'hépatite alcoolique est souhaitable chez les patients avec un score de Maddrey supérieur ou égal à 32 [2,25]. Cela nécessite une ponctionbiopsie hépatique réalisée le plus souvent par voie transjugulaire, en raison des anomalies de la coagulation. Du fait des risques hémorragiques, la pratique d'une biopsie systématique est discutée par certains, même réalisée par voie transjugulaire, car des complications restent possibles comme un hématome au point de ponction jugulaire ou un hémopéritoine dans de rares cas de trajets transcapsulaires sur des foies atrophiques $[2,25]$. Les informations apportées par l'histologie sont d'ordre diagnostique, avec la présence d'une ballonisation hépatocytaire, des corps de Mallory et une infiltration de PNN autour d'hépatocytes en voie de nécrose ou d'apoptose. L'histologie précise également la présence et l'extension de la fibrose, la plupart du temps au stade de cirrhose. Des informations d'ordre pronostique sont également apportées par l'étude histologique. Dans 20 à $30 \%$ des cas, les signes d'hépatite alcoolique ne sont pas trouvés sur la biopsie hépatique de patients avec un score de Maddrey supérieur ou égal 32.

La décision de pratiquer la biopsie hépatique doit aussi tenir compte des disponibilités techniques locales. Les études observationnelles révèlent que seul un tiers des patients avaient réellement une biopsie hépatique. Un diagnostic empirique de l'HAA, prenant en compte l'ensemble du contexte d'alcoolisation et la présentation clinique avec un score de Maddrey supérieur à 32 et l'absence d'autre cause évidente de maladie du foie, est possible avec une bonne précision [28]. Enfin, des alternatives non invasives à la biopsie hépatique ont été étudiées comme l'AshTest ${ }^{\mathbb{B}}$ ou le dosage sérique de la laminine, mais manquent encore de validations indépendantes [29].

\section{Traitements}

Le traitement des patients souffrant d'une HAA nécessite souvent une admission en soins intensifs, pour des mesures réanimatoires, médicamenteuses et un soutien nutritionnel, dans le but de limiter la morbimortalité à court terme. À long terme, la stratégie de prise en charge passe par un suivi hépatologique, mais aussi addictologique en vue d'obtenir une abstinence efficace et prolongée.

\section{Prise en charge addictologique}

La prise en charge addictologique est communément admise comme étant essentielle dans l'HAA [1] ; cependant, peu d'études se sont intéressées à l'évaluer tant elle est implicitement recommandée, alors que dans les faits compte tenu du pronostic vital engagé, la part somatique éclipse souvent la part addictologique dans les premières semaines de la prise en charge médicale. Ainsi, les traitements addictologiques de référence (acamprosate et naltrexone) n'ont pas bénéficié d'évaluation dans l'HAA, même si le baclofène, une ancienne molécule remise au goût de l'addictologie, a été testé dans une étude randomisée en double insu contrôlée versus placebo chez 84 patients cirrhotiques, dont $66 \%$ étaient au stade $\mathrm{C}$ du score de Child-Pugh avec des niveaux maximaux de bilirubine à $56 \mu \mathrm{mol} / 1$ et un INR à 2,7 [30]. Le groupe traité par baclofène pendant 12 semaines avait $71 \%$ de patients abstinents versus $29 \%$ dans le groupe placebo $(\mathrm{OR}=6,3$; IC $95 \%:[2,4-16,1] ; p=0,0001)$, avec une bonne tolérance. Il n'y a pas à ce jour de données chez des patients cirrhotiques Child-Pugh $\mathrm{C}$ avec une HAA sévère.

\section{Prise en charge nutritionnelle}

La supplémentation nutritionnelle est un point essentiel de la stratégie globale de prise en charge de l'HAA sévère. L'apport calorique de l'alcool et les volumes ingérés sont anorexigènes induisant fréquemment une dénutrition. De plus, l'alimentation per os est souvent altérée chez ces patients, ceux consommant moins de $21,5 \mathrm{kcal} / \mathrm{kg}$ par jour ayant un taux de survie plus faible. Les corrections nutritionnelles nécessaires doivent atteindre 1,2 à $1,5 \mathrm{~g}$ de protéines $/ \mathrm{kg}$ et $35-40 \mathrm{kcal} / \mathrm{kg}$ de poids corporel par jour [31]. La voie orale doit être privilégiée, mais l'utilisation d'une nutrition entérale par sonde nasojéjunale est possible, mais mal tolérée, assez souvent enlevée par les patients. La nutrition entérale pourrait participer à une diminution de l'endotoxémie [32].

En dépit du rationnel concernant le support nutritionnel chez les patients ayant une HAA sévère, son impact sur la survie n'est pas bien démontré. L'étude de Cabré et al. n'avait pas montré de différence de survie à j28 avec la nutrition entérale versus les corticostéroïdes, mais la nutrition entérale réduisait la mortalité à long terme ; en revanche, le croisement des courbes de survie appelle à une interprétation prudente [32]. Une étude plus récente montre que la combinaison d'une nutrition entérale intensive par sonde nasogastrique avec des corticostéroïdes n'était pas plus efficace que celle des corticostéroïdes seuls [33]. Dans une analyse de sous-groupe, les patients avec une HAA ayant reçu moins de $21,5 \mathrm{kcal} / \mathrm{kg}$ par jour avaient une mortalité et une fréquence d'infection plus importante à un et six mois [33]. 
Bien qu'il n'y ait pas de bénéfice de survie démontré, la mise en place d'un soutien nutritionnel pour les patients ayant une HAA est recommandée $[1,26]$.

\section{Traitements médicamenteux}

\section{Corticostéroüdes}

Les corticostéroïdes ont été testés dans 15 essais cliniques et 9 méta-analyses [2,3,34-36]. Dans deux méta-analyses les auteurs concluaient sur une inefficacité des corticostéroïdes [34], mais dans l'analyse de sensibilité dans le sous-groupe de patients avec un score de Maddrey supérieur à 32, la survie était améliorée par rapport à celle du groupe placebo [34]. Dans deux méta-analyses à données individuelles [2,3], la survie à court terme à j28 était améliorée par les corticostéroïdes versus le placebo $(79,9 \pm 2,8$ versus $65,7 \pm 3,4 \%, p=0,005)$. Les données de survie à moyen terme sont moins nombreuses et montraient dans une analyse poolée de deux essais une meilleure survie dans le groupe corticostéroïdes à deux mois $(75 \pm 5$ versus $56 \pm 6 \% ; p=0,01)$ et à six mois $(67 \pm 5$ versus $43 \pm 6 \%$, $p<0,01$ ) [2]. Récemment, dans le plus grand essai randomisé en double insu ayant inclus 1106 patients (étude STOPAH), la survie à j28 était améliorée par les corticostéroïdes $(\mathrm{HR}=0,72$; IC $95 \%$ : $[0,52-1,01] ; p=0,06)$, la différence devenant significative après ajustement sur les facteurs pronostiques indépendants (HR $=0,61$; IC $95 \%$ : $[0,41-0,91]$; $p=0,02$ ) [37]. Par contre, il n'y avait aucun bénéfice de survie à trois mois, ni à un an [37]. Cette étude a été incorporée dans une nouvelle méta-analyse à données individuelles, incluant 11 études et 2111 patients [36]. Les résultats indiquaient une meilleure survie à 28 avec les corticostérö̈des $(\mathrm{HR}=0,64 ;$ IC $95 \%:[0,48-0,86] ; p=0,003)$, avec un gain de survie de $7,8 \%$ avec les corticostéroïdes (IC $95 \%$ : $[2,6-12,6] ; \mathrm{NNT}=13)$. À six mois, il n'y avait pas de bénéfice de survie [36]. Ainsi, l'ensemble des données est en faveur d'un gain de survie à j28, mais pas d'effet à moyen et long termes. Les corticostéroïdes sont donc recommandés en traitement de première ligne, avec un grade $\mathrm{A} 1$ selon les guidelines européennes EASL 2018 [1], à la dose de $40 \mathrm{mg} / \mathrm{j}$ de prednisolone ou $32 \mathrm{mg} / \mathrm{j}$ de méthylprednisolone pendant 28 jours, suivi d'un arrêt soit progressif, soit sans décroissance.

\section{Corticostéroïdes plus $\mathrm{N}$-acétylcystéine (bithérapie)}

L'utilisation d'un traitement antioxydant serait justifiée par la présence du stress oxydatif dans l'HAA associant un excès d'espèces ROS et la diminution des capacités antioxydantes de l'hépatocyte. La $N$-acétylcystéine (NAC), par sa capacité à restaurer le niveau de glutathion cellulaire et son action antioxydante, a été testée dans le traitement de l'HAA seule ou en combinaison. En monothérapie, un essai randomisé a été arrêté à l'analyse intermédiaire, car l'utilisation d'un cocktail d'antioxydants (comportant de la NAC) donné pendant 28 jours à la fois par voie orale et en perfusion était significativement inférieure à la prednisolone à $30 \mathrm{mg}$ par jour, pour la survie à un mois [38]. Dans une autre étude, la $N A C$ en perfusion pendant 14 jours plus une nutrition orale et entérale étaient comparées à la nutrition orale et entérale seule. Il n'y avait aucune différence de survie à six mois [39]. Ces deux essais suggèrent que la stratégie antioxydante seule sans corticostéroïdes n'est pas efficace pour traiter l'HAA. Combiner une stratégie antioxydante avec une stratégie anti-inflammatoire aurait l'avantage d'agir à la fois sur les deux phénomènes physiopathologiques, le stress oxydatif et l'excès de TNF- $\alpha$. Dans un essai randomisé, la perfusion de $N A C$ pendant une semaine avec un cocktail antioxydant par voie orale pendant six mois plus corticostéroïdes n'améliorait pas la survie par rapport au groupe placebo plus corticostéroïdes [40], mais cette étude comportait des biais et un manque important de puissance [41]. Dans une autre étude multicentrique française, 174 patients ayant une hépatite alcoolique sévère ont été randomisés entre une combinaison de $N A C$ perfusée pendant cinq jours plus $40 \mathrm{mg}$ de prednisolone par voie orale pendant un mois versus une perfusion de cinq jours de glucosé $5 \%$ plus prednisolone pendant un mois [42]. Dans le groupe des patients traités par la combinaison thérapeutique, la mortalité était significativement moins importante à un mois ( 8 versus $24 \%)[\mathrm{HR}=0,58$; IC $95 \%:[0,14-0,76] ; p=0,006]$, à deux mois $(15,3$ versus $32,6 \% ; p=0,007$; analyse post hoc), mais pas à six mois $(22,4$ versus $33,7 \%)[\mathrm{HR}=0,62$; IC $95 \%:[0,37-1,06]$; $p=0,07]$ qui était l'objectif principal. Le nombre de décès par syndrome hépatorénal était significativement plus faible dans le groupe $N A C+$ corticostéroïdes versus dans le groupe corticostéroïdes seuls ( 9 versus $22 \%$ ) $[\mathrm{OR}=2,79$; IC $95 \%$ : $[1,08-7,42] ; p=0,02]$. Ces résultats étaient en faveur d'un gain de survie précoce avec cette nouvelle stratégie thérapeutique " Corticostéroïdes-NAC », qui a reçu une recommandation européenne EASL 2018 comme traitement de première ligne possible dans l'hépatite alcoolique sévère avec un grade B2 [1].

La NAC associée aux corticostéroïdes est perfusée les cinq premiers jours à la dose de $150 \mathrm{mg} / \mathrm{kg}$ dans $250 \mathrm{ml}$ de glucosé $5 \%$ en 30 à 45 minutes, puis $50 \mathrm{mg} / \mathrm{kg}$ dans $500 \mathrm{ml}$ de G $5 \%$ en quatre heures, puis $100 \mathrm{mg} / \mathrm{kg}$ en 16 heures, la troisième dose étant renouvelée du j2 au j5, puis les corticostéroïdes poursuivis seuls jusqu'à j28 [42]. Ce protocole n'est pas différent de celui utilisé en cas d'intoxication au paracétamol.

\section{Autres traitements non validés}

Parmi les autres traitements, la pentoxifylline est un inhibiteur de la phosphodiestérase et de la synthèse du TNF- $\alpha$. Son 
utilisation dans l'HAA sévère résultait de l'amélioration de la survie intrahospitalière dans un essai randomisé en double insu, avec moins de syndrome hépatorénal [43]. Cependant, les six essais suivants et plusieurs méta-analyses n'ont pas permis de démontrer une supériorité de la pentoxifylline dans l'HAA, que ce soit seule ou associée aux corticostéroïdes versus placebo ou versus corticostéroïdes. [35,36,44]. Avec l'ensemble de ces données, la pentoxifylline n'est plus recommandée selon les guidelines de l'EASL 2018 [1].

Du fait de la constatation de taux élevés de TNF- $\alpha$ dans les modèles animaux et chez les patients avec une HAA sévère, des stratégies de traitement anti-TNF- $\alpha$ ont fait l'objet d'essais cliniques. Malheureusement, tous les essais utilisant un anti-TFN- $\alpha$ (infliximab ou étanercept) seul ou en association aux corticoïdes ont montré un surcroît d'infection ou une surmortalité dans le groupe anti-TNF- $\alpha[45,46]$. Ainsi, les anti-TNF- $\alpha$ seuls ou associés aux corticostérö̈des ne sont pas indiqués dans l'HAA [36].

Une autre stratégie cible la régénération hépatique qui paraît défaillante dans l'HAA sévère [47]. Plusieurs études ont testé le facteur de croissance des granulocytes (G-CSF) connu pour induire l'expression de l'Hepatocyte Grow Factor (HGF) sanguin et intrahépatique [48]. Deux essais thérapeutiques randomisés indiens comparant la combinaison G-CSF plus pentoxifylline versus pentoxifylline seule ont montré une meilleure survie à j90 dans le groupe avec le G-CSF en souscutanée [49-50]. Il faut préciser que l'efficacité de la pentoxifylline est aujourd'hui remise en question. De sorte que lorsque la comparaison a été faite dans une autre étude randomisée avec l'association G-CSF plus transplantation autologue intra-artérielle hépatique de cellules souches plus corticostéroïdes versus corticostéroïdes seuls, l'effet du G-CSF n'a pas été démontré sur la survie à trois mois ni sur la fonction hépatique [51]. Ainsi, l'utilisation du G-CSF dans l'HAA n'est pas indiquée en soins courants [1].

\section{Épurations extrahépatiques}

La dialyse extracorporelle sur colonne d'albumine (système MARS : Molecular Adsorbent Recirculating System) avait donné des résultats préliminaires encourageants sur de petites séries de patients avec une HAA sévère, induisant une diminution de l'hypertension portale sur neuf malades traités [52], une amélioration biologique (baisse de la bilirubine et de la créatinine sérique) et une diminution de l'encéphalopathie hépatique sur huit autres patients avec une HAA [53]. Cependant, dans une série de 19 patients avec une HAA traités par le système MARS versus 19 patients témoins appariés et traités par corticostéroïdes, il n'y avait pas de différence de survie à un et à deux mois [54]. Dans l'étude internationale RELIEF, 189 patients avec une ACLF ont été randomisés pour recevoir un traitement par le système MARS versus un traitement standard. Plus de $81 \%$ des patients avaient une consommation d'alcool excessive ; mais cependant, il n'y avait pas de biopsie hépatique. Des patients avaient potentiellement une hépatite alcoolique non diagnostiquée histologiquement. L'essai n'avait pas démontré de différence de survie avec le système MARS dans la globalité, ni dans des sous-groupes de patients avec une encéphalopathie hépatique, un score de MELD supérieur à 20 ou avec une hyperbilirubinémie progressive [55]. Ces données montrent l'absence probable d'efficacité de la dialyse sur colonne d'albumine avec le système MARS dans le traitement de l'HAA sévère.

Un autre système d'épuration hépatique extracorporelle par des cellules C3A dérivant de lignées d'hépatoblastome (ELAD [Extracorporeal Cellular Therapy]) a été étudié dans une étude randomisée contrôlée internationale [56]. Deux cent trois patients avec une HAA sévère étaient randomisés pour recevoir soit un traitement standard (SOC) plus ELAD pendant 72 à 120 heures $(n=96)$, soit un traitement standard seul $(n=107)$. Il n'y avait pas de différence de survie à j28 (76 versus $80,4 \%$ ) et à $\mathrm{j} 91$ (59,4 versus $61,7 \%)$ entre les groupes SOC plus ELAD et SOC respectivement. Les effets secondaires graves étaient comparables entre les deux groupes. Les résultats de mortalité étaient également non significatifs dans les sous-groupes pré-spécifiés MELD supérieur à $28(68,9$ versus $55,3 \% ; p=0,15)$ ou âge supérieur à 46,9 ans $(60,4$ versus $51 \% ; p=0,26)$. Il y avait une tendance mais non significative à moins de mortalité dans le groupe ELAD pour MELD inférieur à 28 (29,4 versus $43,5 ; p=0,08)$ ou âge inférieur à 46,9 ans (32,6 versus $44,8 \% ; p=0,17)$. Dans une analyse post hoc, le sousgroupe avec les meilleurs critères MELD inférieur à 28 et âge inférieur à 46,9 ans avait un bénéfice du traitement par ELAD avec une survie de 100 versus $75 \%(p=0,006)$. Il faut noter que les patients étaient traités dans moins de $50 \%$ des cas par corticostéroïdes qui est normalement le SOC (standard of care). Il n'y a pas eu d'analyse excluant les patients non traités par corticostéroïdes. Globalement, l'étude est négative, et l'association ELAD plus SOC n'apportant pas de bénéfice évident de survie et ne peut être recommandée dans le traitement des patients avec une HAA.

\section{Réponse au traitement}

Dans une étude rétrospective, la baisse précoce de la bilirubine au septième jour de traitement, définie par une valeur absolue à $\mathrm{j} 7$ numériquement inférieure à celle $\mathrm{du} \mathrm{j} 0$, était associée à une survie de $82,8 \pm 3,3 \%$ à six mois versus seulement $23 \pm 5,8 \%$ en l'absence de baisse [57]. La baisse précoce de la bilirubine au septième jour était dans cette étude un facteur pronostique indépendant de la survie à six mois. Dans une autre étude, les facteurs associés à la survie au sixième mois ont été analysés chez 295 patients avec une hépatite alcoolique sévère [58]. Six variables indépendantes, 
l'âge, l'albuminémie, la bilirubine à j0 et à j7, l'insuffisance rénale et le taux de prothrombine, étaient incorporées dans un score nommé modèle de Lille (http://www.lillemodel. com/score.asp). Avec un cut-off à 0,45 , le modèle de Lille permettait de prédire la survie au sixième mois avec une sensibilité de $76 \%$ et une spécificité de $85 \%$. En utilisant la baisse précoce de la bilirubine à $\mathrm{j} 7$ ou le modèle de Lille inférieur ou égal à $0,45,73,2$ et $62 \%$, des patients respectivement pouvaient être classés comme étant répondeurs au traitement, avec la meilleure survie prévisible. A contrario, les patients non répondeurs étaient définis par l'absence de baisse de la bilirubine à $\mathrm{j} 7$ ou par un modèle de Lille supérieur à 0,45 . Chez ces patients, la survie à six mois était plus faible, mais intéressait tout de même $23 \pm 5,8 \%$ à $25 \pm 3,8 \%$ [59]. Seuls les patients avec un modèle de Lille supérieur à 0,56 ne tiraient aucun bénéfice du traitement par corticostéroïdes par rapport aux mêmes patients non traités par corticostéroïdes. Un modèle de Lille supérieur à 0,56 , correspondant à la réponse nulle, pourrait représenter le cut-off décisionnel pour envisager l'arrêt des corticostéroïdes au septième jour [3]. La prédiction pronostique pourrait être plus robuste en combinant deux méthodes [58]. Ainsi, la combinaison d'un score statique, le MELD avec un score dynamique le modèle de Lille, améliore la prédiction de la mortalité des patients non répondeurs aux corticostéroïdes, en comparaison avec le MELD ou le modèle de Lille seul. Par ailleurs, cette combinaison était supérieure à toutes les autres (Maddrey-Lille; Child-Pugh-Lille).

\section{Traitements de seconde ligne}

En cas de non-réponse aux corticostéroïdes, la mortalité spontanée à six mois est dramatiquement élevée à $75 \%$ [59]. Une étude franco-belge avait réalisé une transplantation hépatique précoce pour 26 patients non répondeurs aux corticostéroïdes avec un score médian de Lille à 0,88 [60]. La survie était significativement meilleure avec $77 \pm 8 \%$ de patients vivants au sixième mois dans le groupe transplantation précoce en comparaison avec seulement $23 \pm 8 \%$ dans le groupe témoin historique apparié et également non répondeur aux corticostéroïdes $(p<0,001)$. La différence était maintenue à deux ans $(71 \pm 9$ versus $23 \pm 8 \% ; p<0,001)$, et le taux de rechute d'alcoolisation était de $11,5 \%$ à deux ans. Du fait de l'impossibilité d'appliquer la règle usuelle de l'abstinence alcoolique de six mois pour une transplantation hépatique précoce, les patients HAA résistant aux corticostéroïdes étaient sélectionnés. Il s'agissait de patients en primo épisode d'HAA, non répondeurs aux corticostéroïdes évalués par le modèle de Lille supérieur à 0,45 à j7, possédant un entourage familial aidant, s'engageant à une abstinence durable, sans comorbidité sévère ni de maladie psychiatrique et ayant donné leur accord. Le processus de décision devait être consensuel entre quatre équipes : une infirmière, un étudiant et un interne en médecine constituaient le premier cercle, le médecin addictologue représentait le deuxième cercle, l'hépatologue senior représentait le troisième cercle, et le médecin anesthésiste et le chirurgien transplanteur représentaient le quatrième cercle. Cette étude a changé l'acceptabilité de la transplantation hépatique précoce pour les patients HAA non répondeurs parmi les membres des équipes de transplantation [61]. Néanmoins, le mode de sélection fait l'objet d'étude de validation en cours, l'accès à un nombre de greffons hépatiques limité faisant entrer en " compétition » d'autres maladies graves du foie non liées à l'alcool ainsi que les cancers du foie. Depuis cette étude, d'autres séries ont été publiées [62], avec des résultats comparables. Dans la cohorte du consortium américain pour la transplantation hépatique précoce des patients HAA résistant aux corticostéroïdes avec une médiane du modèle de Lille à 0,82 , la survie était à $94 \%$ à un an et à $84 \%$ à trois ans [63]. La question de la rechute de la consommation d'alcool posttransplantation est cruciale et a été estimée à $25 \%$ à un an et à $34 \%$ à trois ans, si on prend une définition large " quelle que soit la re-consommation", et à $11 \%$ à un an et à $17 \%$ à trois ans, si on retient une " consommation régulière et soutenue " [64]. Une métaanalyse récente confirme l'ensemble des résultats de survie à $80 \%$ à six mois avec un taux de rechute de consommation d'alcool à $14 \%$ [65]. À l'heure actuelle, les critères de sélection du receveur font encore l'objet d'études qui permettront sans doute bientôt de mieux distinguer les patients les plus à même de bénéficier d'une transplantation hépatique. En attendant, il est important que le réanimateur, face à l'échec de la corticothérapie, se rapproche rapidement d'une équipe d'hépatologues spécialisés dans le greffe hépatique afin d'évaluer l'intérêt d'une transplantation hépatique.

\section{Infections dans l'hépatite alcoolique}

Les infections sont fréquentes chez les patients avec une HAA. Une étude rapporte 25,6\% d'infections constatées dès l'admission à l'hôpital, avant tout traitement (dont 44,4\% d'infections du liquide d'ascite, $12,7 \%$ de pneumopathies, 31,7 \% d'infections urinaires et $11,2 \%$ d'autres sites) [17]. Une méta-analyse rapporte $18,7 \%$ d'infections dans un groupe de patients avec HAA non traitée. L'infection, si elle est contrôlée par antibiotique, ne contre-indique pas formellement les corticostéroïdes. La survie à deux mois était comparable chez les patients infectés et contrôlés par antibiotiques puis traités secondairement par corticostéroïdes par comparaison aux patients non infectés et traités d'emblée par corticostéroïdes $(70,9$ versus $71,6 \% ; p=0,99)$ dans une étude [17]. Une question rémanente est la crainte du risque d'infection pendant le traitement par corticostéroïdes. Dans l'essai STOPAH, les infections survenaient dans $13 \%$ des cas dans le groupe ayant reçu les corticostéroïdes contre $7 \%$ parmi les 
patients sans corticostéroïdes $(p=0,002)$ [37]. Une métaanalyse n'a pas montré de différence d'incidence d'infection bactérienne avec ou sans corticostéroïdes (OR $=0,98$; IC $95 \%$ : [0,49-1,94]) [66]. Dans une autre étude, les infections survenaient dans $23,7 \%$ des cas traités par corticostéroïdes (dont $28,1 \%$ d'infections d'ascite, $40,3 \%$ de pneumopathies, $17,5 \%$ d'infections urinaires et $14,1 \%$ d'autres sites) [17], mais plus que la responsabilité directe des corticostéroïdes, la réponse au traitement avec un modèle de Lille inférieur ou égal à 0,45 était un élément clé avec moins d'infections par rapport aux patients non répondeurs (11,1 versus $42,5 \% ; p<0,000001)$ [17]. Enfin, des infections fungiques ou des pneumocystoses sont également possibles, de diagnostic difficile et survenant chez des patients avec un MELD élevé [67].

\section{Conclusion}

L'HAA sévère doit être reconnue par un score de Maddrey supérieur ou égal à 32 et au mieux authentifiée par une histologie hépatique obtenue par voie transjugulaire. Les infections bactériennes, très fréquentes dans ce contexte, doivent être dépistées systématiquement avant le traitement. Les corticostéroïdes représentent le traitement de première ligne, associés à un soutien nutritionnel adapté et à une abstinence alcoolique. L'association corticostéroïdes plus $N A C$ améliore la survie à court terme par rapport aux corticostéroïdes seuls, constituant une option thérapeutique de première ligne. La réponse au traitement est évaluée au septième jour par la baisse de la bilirubine ou un modèle de Lille inférieur à 0,45 . Chez les patients non répondeurs aux corticostéroïdes, la transplantation hépatique précoce, chez des patients sélectionnés, améliore la survie significativement.

Liens d'intérêts : les auteurs déclarent ne pas avoir de lien d'intérêt.

\section{Références}

1. European Association for the Study of the Liver, (2018) EASL Clinical Practice Guidelines: management of alcohol-related liver disease. J Hepatol 69: 154-181

2. Mathurin P, Mendenhall CL, Carithers RL, Ramond MJ, Maddrey WC, Garstide P, Rueff B, Naveau S, Chaput JC, Poynard T, (2002) Corticosteroids improve short-term survival in patients with severe alcoholic hepatitis (AH): individual data analysis of the last three randomized placebo controlled double blind trials of corticosteroids in severe AH. J Hepatol 36: 480-487

3. Mathurin P, O'Grady J, Carithers RL, Phillips M, Louvet A, Mendenhall CL, Ramond MJ, Naveau S, Maddrey WC, Morgan TR, (2011) Corticosteroids improve short-term survival in patients with severe alcoholic hepatitis: meta-analysis of individual patient data. Gut 60: 255-260
4. Ciocan D, Rebours V, Voican CS, Wrzosek L, Puchois V, Cassard AM, Perlemuter G, (2018) Characterization of intestinal microbiota in alcoholic patients with and without alcoholic hepatitis or chronic alcoholic pancreatitis. Sci Rep 8: 4822

5. Llopis M, Cassard AM, Wrzosek L, Boschat L, Bruneau A, Ferrere G, Puchois V, Martin JC, Lepage P, Le Roy T, Lefèvre L, Langelier B, Cailleux F, González-Castro AM, Rabot S, Gaudin F, Agostini H, Prévot S, Berrebi D, Ciocan D, Jousse C, Naveau S, Gérard P, Perlemuter G, (2016) Intestinal microbiota contributes to individual susceptibility to alcoholic liver disease. Gut 65 : 830-839

6. Albano E, Clot P, Morimoto M, Tomasi A, Ingelman-Sundberg M, French SW, (1996) Role of cytochrome P4502E1-dependent formation of hydroxyethyl free radical in the development of liver damage in rats intragastrically fed with ethanol. Hepatology 23: $155-163$

7. Hirano T, Kaplowitz N, Tsukamoto H, Kamimura S, FernandezCheca JC, (1992) Hepatic mitochondrial glutathione depletion and progression of experimental alcoholic liver disease in rats. Hepatology 16: 1423-1427

8. Oh SI, Kim CI, Chun HJ, Park SC, (1998) Chronic ethanol consumption affects glutathione status in rat liver. J Nutr 128: 758-763

9. Lands WE, (1995) Cellular signals in alcohol-induced liver injury: a review. Alcohol Clin Exp Res 19: 928-938

10. Colell A, García-Ruiz C, Miranda M, Ardite E, Marí M, Morales A, Corrales F, Kaplowitz N, Fernández-Checa JC, (1998) Selective glutathione depletion of mitochondria by ethanol sensitizes hepatocytes to tumor necrosis factor. Gastroenterology 115: $1541-1551$

11. Adachi Y, Bradford BU, Gao W, Bojes HK, Thurman RG, (1994) Inactivation of Kupffer cells prevents early alcohol-induced liver injury. Hepatology 20: 453-460

12. Mathurin P, Deng QG, Keshavarzian A, Choudhary S, Holmes EW, Tsukamoto H, (2000) Exacerbation of alcoholic liver injury by enteral endotoxin in rats. Hepatology 32: 1008-1017

13. Pritchard MT, McMullen MR, Stavitsky AB, Cohen JI, Lin F, Edward Medof M, Nagy LE, (2007) Differential contributions of $\mathrm{C} 3, \mathrm{C} 5$, and decay-accelerating factor to ethanol-induced fatty liver in mice. Gastroenterology 132: 1117-1126

14. Miller AM, Horiguchi N, Jeong WI, Radaeva S, Gao B, (2011) Molecular mechanisms of alcoholic liver disease: innate immunity and cytokines. Alcohol Clin Exp Res 35: 787-793

15. Hrubec Z, Omenn GS, (1981) Evidence of genetic predisposition to alcoholic cirrhosis and psychosis: twin concordances for alcoholism and its biological end points by zygosity among male veterans. Alcohol Clin Exp Res 5: 207-215

16. Nguyen-Khac E, Houchi H, Daoust M, Dupas JL, Naassila M, (2008) The -308 TNFalpha gene polymorphism in severe acute alcoholic hepatitis: identification of a new susceptibility marker. Alcohol Clin Exp Res 32: 822-828

17. Louvet A, Wartel F, Castel H, Dharancy S, Hollebecque A, CanvaDelcambre V, Deltenre P, Mathurin P, (2009) Infection in patients with severe alcoholic hepatitis treated with steroids: early response to therapy is the key factor. Gastroenterology 137: 541-548

18. Maddrey WC, Boitnott JK, Bedine MS, Weber FL, Mezey E, White RI, (1978) Corticosteroid therapy of alcoholic hepatitis. Gastroenterology 75: 193-199

19. Carithers RL, Herlong HF, Diehl AM, Shaw EW, Combes B, Fallon HJ, Maddrey WC, (1989) Methylprednisolone therapy in patients with severe alcoholic hepatitis. A randomized multicenter trial. Ann Intern Med 110: 685-690

20. Sheth M, Riggs M, Patel T, (2002) Utility of the Mayo End-Stage Liver Disease (MELD) score in assessing prognosis of patients with alcoholic hepatitis. BMC Gastroenterol 2: 2 
21. Soultati AS, Dourakis SP, Alexopoulou A, Deutsch M, Vasilieva L, Archimandritis AJ, (2006) Predicting utility of a model for end stage liver disease in alcoholic liver disease. World J Gastroenterol 12: 4020-4025

22. Forrest EH, Morris AJ, Stewart S, Phillips M, Oo YH, Fisher NC, Haydon G, O'Grady J, Day CP, (2007) The Glasgow alcoholic hepatitis score identifies patients who may benefit from corticosteroids. Gut 56:1743-1746

23. Dominguez M, Rincón D, Abraldes JG, Miquel R, Colmenero J, Bellot P, García-Pagán JC, Fernández R, Moreno M, Bañares R, Arroyo V, Caballería J, Ginès P, Bataller R, (2008) A new scoring system for prognostic stratification of patients with alcoholic hepatitis. Am J Gastroenterol 103: 2747-2756

24. EH Forrest, NC Fisher, S Singhal, A Brind, G Haydon, J O'Grady, PC Hayes, CP Day, (2010) Comparison of the Glasgow alcoholic hepatitis score and the ABIC score for the assessment of alcoholic hepatitis. Am J Gastroenterol 105: 701-702 (doi: 10.1038/ajg.2009.645)

25. O'Shea RS, Dasarathy S, McCullough AJ, Practice Parameters Committee of the American College of Gastroenterology, (2010) Alcoholic liver disease. Hepatology 51: 307-328

26. Moreau R, Jalan R, Gines P, Pavesi M, Angeli P, Cordoba J, Durand F, Gustot T, Saliba F, Domenicali M, Gerbes A, Wendon J, Alessandria C, Laleman W, Zeuzem S, Trebicka J, Bernardi M, Arroyo V; CANONIC Study Investigators of the EASL-CLIF Consortium, (2013) Acute-on-chronic liver failure is a distinct syndrome that develops in patients with acute decompensation of cirrhosis. Gastroenterology 144: 1426-1437, 1437.e1-9

27. Sersté T, Cornillie A, Njimi H, Pavesi M, Arroyo V, Putignano A, Weichselbaum L, Deltenre P, Degré D, Trépo E, Moreno C, Gustot T, (2018) The prognostic value of acute-on-chronic liver failure during the course of severe alcoholic hepatitis. J Hepatol 69: 318-324

28. Kryger P, Schlichting P, Dietrichson O, Juhl E, (1983) The accuracy of the clinical diagnosis in acute hepatitis and alcoholic liver disease. Clinical versus morphological diagnosis. Scand J Gastroenterol 18: 691-6

29. Thabut D, Naveau S, Charlotte F, Massard J, Ratziu V, ImbertBismut F, Cazals-Hatem D, Abella A, Messous D, Beuzen F, Munteanu M, Taieb J, Moreau R, Lebrec D, Poynard T, (2006) The diagnostic value of biomarkers (AshTest ${ }^{\mathbb{R}}$ ) for the prediction of alcoholic steato-hepatitis in patients with chronic alcoholic liver disease. J Hepatol 44: 1175-1185

30. Addolorato G, Leggio L, Ferrulli A, Cardone S, Vonghia L, Mirijello A, Abenavoli L, D'Angelo C, Caputo F, Zambon A, Haber PS, Gasbarrini G, (2007) Effectiveness and safety of baclofen for maintenance of alcohol abstinence in alcohol-dependent patients with liver cirrhosis: randomised, double-blind controlled study. Lancet 370: 1915-1922

31. Plauth M, Cabré E, Riggio O, Assis-Camilo M, Pirlich M, Kondrup J, Ferenci P, Holm E, Vom Dahl S, Müller MJ, Nolte W; ESPEN (European Society for Parenteral and Enteral Nutrition), (2006) ESPEN guidelines on enteral nutrition: liver disease. Clin Nutr 25: 285-294

32. Cabré E, Rodríguez-Iglesias P, Caballería J, Quer JC, SánchezLombraña JL, Parés A, Papo M, Planas R, Gassull MA, (2000) Short- and long-term outcome of severe alcohol-induced hepatitis treated with steroids or enteral nutrition: a multicenter randomized trial. Hepatology 32: 36-42

33. Moreno C, Deltenre P, Senterre C, Louvet A, Gustot T, Bastens B, Hittelet A, Piquet MA, Laleman W, Orlent H, Lasser L, Sersté T, Starkel P, De Koninck X, Negrin Dastis S, Delwaide J, Colle I, de Galocsy C, Francque S, Langlet P, Putzeys V, Reynaert H, Degré D, Trépo E, (2016) Intensive Enteral Nutrition Is Ineffective for Patients With Severe Alcoholic Hepatitis Treated With Corticosteroids. Gastroenterology 150: 903-910.e8
34. Rambaldi A, Saconato HH, Christensen E, Thorlund K, Wetterslev J, Gluud C, (2008) Systematic review: glucocorticosteroids for alcoholic hepatitis - a Cochrane Hepato-Biliary Group systematic review with meta-analyses and trial sequential analyses of randomized clinical trials. Aliment Pharmacol Ther 27: 1167-1178

35. Singh S, Murad MH, Chandar AK, Bongiorno CM, Singal AK, Atkinson SR, Thursz MR, Loomba R, Shah VH, (2015) Comparative effectiveness of pharmacological interventions for severe alcoholic hepatitis: a systematic review and network metaanalysis. Gastroenterology 149: 958-970.e12

36. Louvet A, Thursz MR, Kim DJ, Labreuche J, Atkinson SR, Sidhu SS, O'Grady JG, Akriviadis E, Sinakos E, Carithers RL, Ramond MJ, Maddrey WC, Morgan TR, Duhamel A, Mathurin P, (2018) Corticosteroids reduce risk of death within 28 days for patients with severe alcoholic hepatitis, compared with pentoxifylline or placebo-a meta-analysis of individual data from controlled trials. Gastroenterology 155: 458-468.e8

37. Thursz MR, Richardson P, Allison M, Austin A, Bowers M, Day CP, Downs N, Gleeson D, MacGilchrist A, Grant A, Hood S, Masson S, McCune A, Mellor J, O'Grady J, Patch D, Ratcliffe I, Roderick P, Stanton L, Vergis N, Wright M, Ryder S, Forrest EH; STOPAH Trial, (2015) Prednisolone or pentoxifylline for alcoholic hepatitis. N Engl J Med 372: 1619-1628

38. Phillips M, Curtis H, Portmann B, Donaldson N, Bomford A, O'Grady J, (2006) Antioxidants versus corticosteroids in the treatment of severe alcoholic hepatitis - a randomised clinical trial. J Hepatol 44: 784-790

39. Moreno C, Langlet P, Hittelet A, Lasser L, Degré D, Evrard S, Colle I, Lemmers A, Devière J, Le Moine O, (2010) Enteral nutrition with or without $N$-acetylcysteine in the treatment of severe acute alcoholic hepatitis: a randomized multicenter controlled trial. J Hepatol 53: 1117-1122

40. Stewart S, Prince M, Bassendine M, Hudson M, James O, Jones D, Record C, Day CP, (2007) A randomized trial of antioxidant therapy alone or with corticosteroids in acute alcoholic hepatitis. J Hepatol 47: 277-283

41. Nguyen-Khac E, ThevenoT t, Piquet MA, Benferhat S, Hezam A, Goria O, Tramier B, Chatelain D, Dupas JL, (2008) Antioxidants plus corticosteroids in the treatment of severe acute alcoholic hepatitis: the question is still open. J Hepatol 49: 147-148 (author reply 148. doi: 10.1016/j.jhep.2008.04.003. Epub 2008 Apr 28)

42. Nguyen-Khac E, Thevenot T, Piquet MA, Benferhat S, Goria O, Chatelain D, Tramier B, Dewaele F, Ghrib S, Rudler M, Carbonell N, Tossou H, Bental A, Bernard-Chabert B, Dupas JL; AAHNAC Study Group, (2011) Glucocorticoids plus $N$-acetylcysteine in severe alcoholic hepatitis. N Engl J Med 365: 1781-1789

43. Akriviadis E, Botla R, Briggs W, Han S, Reynolds T, Shakil O, (2000) Pentoxifylline improves short-term survival in severe acute alcoholic hepatitis: a double-blind, placebo-controlled trial. Gastroenterology 119: 1637-1648

44. Whitfield K, Rambaldi A, Wetterslev J, Gluud C, (2009) Pentoxifylline for alcoholic hepatitis. Cochrane Database Syst Rev 4: CD007339 (doi: 10.1002/14651858.CD007339.pub2)

45. Naveau S, Chollet-Martin S, Dharancy S, Mathurin P, Jouet P, Piquet MA, Davion T, Oberti F, Broët P, Emilie D; Foie-Alcool group of the Association française pour l'étude du foie, (2004) A double-blind randomized controlled trial of infliximab associated with prednisolone in acute alcoholic hepatitis. Hepatology 39: 1390-1397

46. Boetticher NC, Peine CJ, Kwo P, Abrams GA, Patel T, Aqel B, Boardman L, Gores GJ, Harmsen WS, McClain CJ, Kamath PS, Shah VH, (2008) A randomized, double-blinded, placebocontrolled multicenter trial of etanercept in the treatment of alcoholic hepatitis. Gastroenterology 135: 1953-1960 
47. Dubuquoy L, Louvet A, Lassailly G, Truant S, Boleslawski E, Artru F, Maggiotto F, Gantier E, Buob D, Leteurtre E, Cannesson A, Dharancy S, Moreno C, Pruvot FR, Bataller R, Mathurin P, (2015) Progenitor cell expansion and impaired hepatocyte regeneration in explanted livers from alcoholic hepatitis. Gut 64: 1949-1960

48. Spahr L, Lambert JF, Rubbia-Brandt L, Chalandon Y, Frossard JL, Giostra E, Hadengue A, (2008) Granulocyte-colony stimulating factor induces proliferation of hepatic progenitors in alcoholic steatohepatitis: a randomized trial. Hepatology 48: 221-229

49. Singh V, Sharma AK, Narasimhan RL, Bhalla A, Sharma N, Sharma R, (2014) Granulocyte colony-stimulating factor in severe alcoholic hepatitis: a randomized pilot study. Am J Gastroenterol 109: 1417-1423

50. Singh V, Keisham A, Bhalla A, Sharma N, Agarwal R, Sharma R, Singh A, (2018) Efficacy of granulocyte colony-stimulating factor and $\mathrm{N}$-acetylcysteine therapies in patients with severe alcoholic hepatitis. Clin Gastroenterol Hepatol 16: 1650-1656.e2 (doi: 10.1016/j.cgh.2018.01.040. Epub 2018 Jan 31)

51. Spahr L, Chalandon Y, Terraz S, Kindler V, Rubbia-Brandt L, Frossard JL, Breguet R, Lanthier N, Farina A, Passweg J, Becker CD, Hadengue A, (2013) Autologous bone marrow mononuclear cell transplantation in patients with decompensated alcoholic liver disease: a randomized controlled trial. PLoS One 8: e53719

52. Sen S, Mookerjee RP, Cheshire LM, Davies NA, Williams R, Jalan R, (2005) Albumin dialysis reduces portal pressure acutely in patients with severe alcoholic hepatitis. J Hepatol 43: 142-148

53. Jalan R, Sen S, Steiner C, Kapoor D, Alisa A, Williams R, (2003) Extracorporeal liver support with molecular adsorbents recirculating system in patients with severe acute alcoholic hepatitis. J Hepatol 38: 24-31

54. Boitard J, Louvet A, Bismuth B, Dharancy S, Wartel F, CanvaDelcambre V, Deltenre P, Jude B, Fourrier F, Mathurin P, (2008) Tolerance and efficacy of the mars system in patients with severe alcoholic hepatitis non-responders to steroids: a pilot study. J Hepatol 48: s355-s356

55. Bañares R, Nevens F, Larsen FS, Jalan R, Albillos A, Dollinger M, Saliba F, Sauerbruch T, Klammt S, Ockenga J, Pares A, Wendon J, Brünnler T, Kramer L, Mathurin P, de la Mata M, Gasbarrini A, Müllhaupt B, Wilmer A, Laleman W, Eefsen M, Sen S, Zipprich A, Tenorio T, Pavesi M, Schmidt HH, Mitzner S, Williams R, Arroyo V; RELIEF study group, (2013) Extracorporeal albumin dialysis with the molecular adsorbent recirculating system in acute-on-chronic liver failure: the RELIEF trial. Hepatology 57: 1153-1162

56. Thompson J, Jones N, Al-Khafaji A, Malik S, Reich D, Munoz S, MacNicholas R, Hassanein T, Teperman L, Stein L, DuarteRojo A, Malik R, Adhami T, Asrani S, Shah N, Gaglio P, Duddempudi A, Borg B, Jalan R, Brown R, Patton H, Satoskar R, Rossi S, Parikh A, ElSharkawy A, Mantry P, Sher L, Wolf D, Hart M, Landis C, Wigg A, Habib S, McCaughan G, Colquhoun S, Henry A, Bedard P, Landeen L, Millis M, Ashley R, Frank W, Henry A, Stange J, Subramanian R, VTI-208 Study Group, (2018) Extracorporeal Cellular Therapy (ELAD) in severe alcoholic hepatitis: a multinational, prospective, controlled, randomized trial. Liver Transpl 24: 380-393
57. Mathurin P, Abdelnour M, Ramond MJ, Carbonell N, Fartoux L, Serfaty L, Valla D, Poupon R, Chaput JC, Naveau S, (2003) Early change in bilirubin levels is an important prognostic factor in severe alcoholic hepatitis treated with prednisolone. Hepatology 38: 1363-1369

58. Louvet A, Labreuche J, Artru F, Boursier J, Kim DJ, O'Grady J, Trépo E, Nahon P, Ganne-Carrié N, Naveau S, Diaz E, Gustot T, Lassailly G, Cannesson-Leroy A, Canva-Delcambre V, Dharancy S, Park SH, Moreno C, Morgan TR, Duhamel A, Mathurin P, (2015) Combining Data From Liver Disease Scoring Systems Better Predicts Outcomes of Patients With Alcoholic Hepatitis. Gastroenterology 149: 398-406.e8 (quiz e16-7)

59. Louvet A, Naveau S, Abdelnour M, Ramond MJ, Diaz E, Fartoux L, Dharancy S, Texier F, Hollebecque A, Serfaty L, Boleslawski E, Deltenre P, Canva V, Pruvot FR, Mathurin P, (2007) The Lille model: a new tool for therapeutic strategy in patients with severe alcoholic hepatitis treated with steroids. Hepatology 45: $1348-1354$

60. Mathurin P, Moreno C, Samuel D, Dumortier J, Salleron J, Durand F, Castel H, Duhamel A, Pageaux GP, Leroy V, Dharancy S, Louvet A, Boleslawski E, Lucidi V, Gustot T, Francoz C, Letoublon C, Castaing D, Belghiti J, Donckier V, Pruvot FR, Duclos-Vallée JC, (2011) Early liver transplantation for severe alcoholic hepatitis. N Engl J Med 365: 1790-1800

61. TM Antonini, O Guillaud, J Dumortier, S Dharancy, F Saliba, P Mathurin, JC Duclos-Vallée, C Duvoux; for Groupe de recherche français en greffe de foie (GReF2), (2018) Impact of a first study of early transplantation in acute alcoholic hepatitis: results of a nationwide survey in french liver transplantation programs. Liver Transpl 24: 841-844 (doi: 10.1002/lt.25039. Epub 2018 May 13)

62. Im GY, Kim-Schluger L, Shenoy A, Schubert E, Goel A, Friedman SL, Florman S, Schiano TD, (2016) Early liver transplantation for severe alcoholic hepatitis in the United States - a Single-Center Experience. Am J Transplant 16: 841-849

63. Lee BP, Chen PH, Haugen C, Hernaez R, Gurakar A, Philosophe B, Dagher N, Moore SA, Li Z, Cameron AM, (2017) Three-year results of a pilot program in early liver transplantation for severe alcoholic hepatitis. Ann Surg 265: 20-29

64. Lee BP, Mehta N, Platt L, Gurakar A, Rice JP, Lucey MR, Im GY, Therapondos G, Han H, Victor DW, Fix OK, Dinges L, Dronamraju D, Hsu C, Voigt MD, Rinella ME, Maddur H, Eswaran S, Hause J, Foley D, Ghobrial RM, Dodge JL, Li Z, Terrault NA, (2018) Outcomes of early liver transplantation for patients with severe alcoholic hepatitis. Gastroenterology 155: 422-430.e1

65. Marot A, Dubois M, Trépo E, Moreno C, Deltenre P, (2018) Liver transplantation for alcoholic hepatitis: a systematic review with meta-analysis. PLoS One 13: e0190823

66. Hmoud BS, Patel K, Bataller R, Singal AK, (2016) Corticosteroids and occurrence of and mortality from infections in severe alcoholic hepatitis: a meta-analysis of randomized trials. Liver Int 36: 721-728

67. Gustot T, Maillart E, Bocci M, Surin R, Trépo E, Degré D, Lucidi V, Taccone FS, Delforge ML, Vincent JL, Donckier V, Jacobs F, Moreno C, (2014) Invasive aspergillosis in patients with severe alcoholic hepatitis. J Hepatol 60: 267-274 